\title{
The Construction of Movement with Behavior-Specific and Behavior-Independent Modules
}

\author{
Jian Jing, Elizabeth C. Cropper, Itay Hurwitz, and Klaudiusz R. Weiss \\ Department of Physiology and Biophysics, Mount Sinai School of Medicine, New York, New York 10029-6574
}

Growing evidence suggests that different forms of complex motor acts are constructed through flexible combinations of a small number of modules in interneuronal networks. It remains to be established, however, whether a module simply controls groups of muscles and functions as a computational unit for use in multiple behaviors (behavior independent) or whether a module controls multiple salient features that define one behavior and is used primarily for that behavior (behavior specific). We used the Aplysia feeding motor network to examine the two proposals by studying the functions of identifiable interneurons. We identified three types of motor programs that resemble three types of behaviors that Aplysia produce: biting, swallowing, and rejection. Two ingestive programs (biting, swallowing) are defined by two movement parameters of the feeding apparatus (the radula): one is the same in both programs (phasing of radula closure motoneurons relative to radula protraction-retraction), whereas the other parameter (protraction duration) is different in the two programs. In each program, these two parameters were specified together by an individual neuron, but the neurons in each were different (B40 for biting, B30 for swallowing). These findings support the existence of behavior-specific modules. Furthermore, neuron B51 was found to mediate a phase that can be flexibly added on to both ingestive and egestive-rejection programs, suggesting that B51 may be a behavior-independent module. The functional interpretation of the role played by these modules is supported by the patterns of synaptic connectivity that they make. Thus, both behavior-specific and behavior-independent modules are used to construct complex behaviors.

Key words: module; modular organization; Aplysia; feeding; spinal system; motor programs; movement parameter coding; interneuron; central pattern generator

\section{Introduction}

To produce multiple forms of structured motor acts, animals commonly use a single motor apparatus, such as a limb or a feeding apparatus. Because these peripheral structures are typically controlled by multiple sets of agonist and antagonist muscles, the major challenge for students of motor control is to explain how activity in the nervous systems is coordinated so that functional movements are generated (Bernstein, 1967). Work in both vertebrates and invertebrates suggests that distinct behaviors are constructed through flexible combinations of a small number of building blocks or modules (Grillner, 1981; Bizzi et al., 1991; Stein and Smith, 1997; Jing and Weiss, 2002; Tresch et al., 2002); however, it is poorly understood what constitutes a module and whether there are different types of modules. The resolution of these issues requires a direct examination of specific functions of relevant interneurons or interneuronal populations that presumably serve as modules. In vertebrates, a module is often

Received Jan. 29, 2004; revised June 1, 2004; accepted June 2, 2004

This work was supported by grants from the National Institute of Mental Health (R01 MH50235, K05 MH 01427, and F32 MH12890). We thank Alex Proekt for assistance in data analysis.

Correspondence should be addressed to Dr. Jian Jing, Department of Physiology and Biophysics, Box 1218, Mount Sinai School of Medicine, 1 Gustave Levy Place, New York, NY 10029. E-mail: jjing@inka.mssm.edu.

I. Hurwitz's present address: Interdisciplinary Program in the Brain Sciences, Bar-llan University, Ramat Gan 52900, Israel.

DOI:10.1523/JNEUROSCI.0965-04.2004

Copyright $\odot 2004$ Society for Neuroscience $\quad$ 0270-6474/04/246315-11\$15.00/0 composed of a population of interneurons, whereas in invertebrates, single interneurons may function as a module. We are studying identifiable interneurons in a small motor network in Aplysia that mediate feeding to examine issues relevant to modular organization (Jing and Weiss, 2002).

The modular concept was originally proposed to explain flexibility in coordination patterns of vertebrate limbs during diverse behaviors (Grillner, 1981, 1985; Jordan, 1991; Stein et al., 1995). It refers to the control of groups of agonist muscles, e.g., around a joint, by a small number of interneurons or groups of functionally related interneurons. Data that support the modular concept have been obtained in studies of the motor control of frog limbs (Tresch et al., 1999; d'Avella et al., 2003) and turtle scratching (Stein and Daniels-McQueen, 2002); however, outstanding issues remain. Specifically, two schemes concerning composition and function of modules have been proposed (Bernstein, 1967; Tresch et al., 2002). A module may reduce the number of degrees of freedom by controlling groups of muscles and function as a computational unit for use in multiple, but not necessarily all, behaviors (i.e., be behavior independent). Alternatively, a module may control the multiple salient features that define a behavior and therefore may be used primarily for that behavior (i.e., be behavior specific).

Like the vertebrate spinal system (Mortin et al., 1985; Grasso et al., 1998), the Aplysia feeding network produces multiple forms of related behaviors: biting, swallowing, and rejection 
(Kupfermann, 1974; Morton and Chiel, 1993a). Previous work on biting and rejection-like motor programs (Jing and Weiss, 2001, 2002; Jing et al., 2003) suggested that the Aplysia feeding network has a modular organization. Here, we characterize swallowing and rejection-like motor programs that are elicited by a higher-order interneuron, cerebral-buccal interneuron-4 (CBI4 ), and determine whether modules are behavior specific or independent. We find that biting and swallowing-like programs are generated by different modules, despite the fact that biting and swallowing-like programs share one parameter (phasing of closure motoneurons relative to protraction-retraction). This supports the idea that modules are behavior specific. Furthermore, CBI-4-elicited swallowing and rejection-like programs have a phase that can be flexibly added on. This phase is mediated by a behavior-independent module. Thus, different but related behaviors are constructed through combinations of behaviorspecific and behavior-independent modules.

\section{Materials and Methods}

Specimens of Aplysia californica were obtained from Marinus (Long Beach, CA) and from the National Resource for Aplysia at the University of Miami. They were maintained in circulating artificial seawater (ASW), made from Instant Ocean (Aquarium Systems, Mentor, $\mathrm{OH}$ ) at $14-15^{\circ} \mathrm{C}$. Animals weighing $50-250$ gm were anesthetized by injection ( $50 \%$ of the body weight) of isotonic $\mathrm{MgCl}_{2}$ (337 mM). Cerebral ganglia together with buccal ganglia were dissected out and desheathed. Ganglia were then pinned in a chamber that had a volume of $\sim 1.5 \mathrm{ml}$. The preparation was continuously perfused with ASW containing (in $\mathrm{mm}$ ): $460 \mathrm{NaCl}, 10 \mathrm{KCl}, 55 \mathrm{MgCl}_{2}, 11 \mathrm{CaCl}_{2}$, and 10 HEPES buffer, $\mathrm{pH} 7.6$, at a rate of $0.3 \mathrm{ml} / \mathrm{min}$, and maintained at $14-17^{\circ} \mathrm{C}$. All chemicals were purchased from Sigma (St. Louis, MO).

Standard intracellular and extracellular recordings were obtained as described (Jing and Weiss, 2001). Digitized data were plotted with Axum (Mathsoft, Cambridge, MA). Functional synaptic connections were examined in normal saline (ASW), and the ability of PSPs to follow presynaptic spikes one-for-one was taken as an indication of probable monosynapticity. Assays of monosynapticity were conducted in high-divalent saline containing (in $\mathrm{mm}$ ): $312 \mathrm{NaCl}, 10 \mathrm{KCl}, 132 \mathrm{MgCl}_{2}, 33 \mathrm{CaCl}_{2}$, and 10 HEPES, pH 7.6, a solution that elevates spike thresholds and thus curtails polysynaptic activation (Cohen et al., 1978; Trudeau and Castellucci, 1992). Neurons were identified on the basis of location, size, and electrophysiological and morphological characteristics (Rosen et al., 1991; Hurwitz et al., 1997; Jing and Weiss, 2001; Jing et al., 2003).

Motor programs were elicited by stimulation of higher-order interneurons, i.e., CBI-2 or CBI-4. For each cycle of a motor program, the protraction phase was monitored by the activity in the $\mathrm{I} 2$ nerve, which contains axons of protraction motoneurons B31/32 and B61/62 (Hurwitz et al., 1996). The retraction phase was monitored by sustained depolarization in $\mathrm{B} 4 / 5$ after protraction or hyperpolarization in protraction interneurons-motoneurons (Jing and Weiss, 2001, 2002). Radula closure was monitored by recording from the radula nerve ( $\mathrm{RN})$, which contains axons of the radula closure motoneuron B8, or by recording intracellularly from B8 (Morton and Chiel, 1993a,b). The RN contains axons of neurons other than $\mathrm{B} 8$, but all of the large units recorded in this nerve originate from the B8 motoneurons (Morton and Chiel, 1993b).

Aplysia feeding behaviors are stimulus driven (Kupfermann, 1974), i.e., they can occur both in single or multiple cycles, depending on the duration of contact with food. Similarly, in the isolated nervous system, depending on the duration of stimulation of the CBIs, buccal ganglia produce either single or multiple cycles of activity, as shown previously for both CBI-2 and CBI-4 (Rosen et al., 1991; Jing and Weiss, 2001; Morgan et al., 2002). When multiple cycles of a motor program are elicited by stimulation of CBI-2 or CBI-4, individual cycles have characteristics that are similar to those that are elicited repetitively in single cycles. Essentially for technical reasons, several recent papers (Jing and Weiss, 2001, 2002; Dembrow et al., 2003; Jing et al., 2003) have used the single-cycle paradigm. Single-cycle motor programs elicited by stimulation of the CBIs are more reproducible and therefore can be used to more accurately study effects induced by manipulation of specific interneurons or neurotransmitters. For these reasons, we have adopted the single-cycle paradigm for most of our experiments, in which the frequency of stimulation of CBIs remains similar in different episodes. Because stimulation of CBIs beyond the end of protraction does not affect the parameters of motor programs (Jing et al., 2003), in some cases, stimulation was stopped when protraction ended.

Throughout Results, $n$ refers to the number of preparations, except for data shown in Figures 2 and 3 and data for B40 and B30 activity during CBI-4-elicited programs, where $n$ refers to the number of observations and the data were obtained from a dataset of 26 preparations. Data are expressed as mean \pm SEM.

\section{Results}

\section{Movement parameters that define the types of feeding motor programs}

There are at least three functionally effective forms of feedingrelated behaviors: biting, swallowing, and rejection (Kupfermann, 1974). They are defined by a set of movement parameters (Fig. $1 A$ ) of the feeding apparatus, the radula (Morton and Chiel, 1993a; Jing and Weiss, 2002). Radula movements involve alternating protraction-retraction movements that are coupled with opening-closing movements. In all three behaviors, protraction precedes retraction, and both are necessary movements, regardless of the type of behavior, because they serve to move the radula forward and backward in a rhythmic manner. Biting is triggered by external stimulation of the mouth, whereas swallowing is triggered by the presence of food in the buccal cavity (Kupfermann, 1974). Biting and swallowing are ingestive behaviors and can be distinguished from egestion-rejection by the phasing of radula closing relative to protraction-retraction. Thus, in ingestion, closing occurs during retraction to facilitate food grasping and intake, whereas in egestion, closing occurs during protraction to push an inedible object out of the mouth. Furthermore, biting can be distinguished from swallowing by the length of protraction (Hurwitz et al., 1996). During biting, protraction is prolonged to enable an animal to contact food outside of the mouth; during swallowing, protraction is short because the food is, at least partially, inside the mouth. Not all responses are effective or functional because they are not accompanied by a clear difference in the opening-closure state of the radula during protraction and retraction (Morton and Chiel, 1993a). These responses may be a manifestation of what has been referred to as intermediate motor programs (Morton and Chiel, 1993b; Morgan et al., 2002).

Fictive motor programs (Fig. $1 A$ ) resembling feeding-related behaviors can be elicited in the isolated CNS by stimulation of CBIs (Rosen et al., 1991). Motor programs elicited by the command-like neuron CBI-2 have been studied most extensively (Church and Lloyd, 1994; Jing and Weiss, 2001; Morgan et al., 2002; Hurwitz et al., 2003). Recent work has suggested that CBI-2 can elicit both ingestive and egestive motor programs and that ingestive activity resembles biting because protraction is relatively long (Jing and Weiss, 2001, 2002; Morgan et al., 2002; Jing et al., 2003). In addition, CBI-2 can also elicit intermediate motor programs in which closure motoneurons are moderately active during both protraction and retraction (Morgan et al., 2002). The interneuronal circuit that is activated by stimulation of CBI-2 is depicted in Figure $1 B$ (left panel). Before this study, the swallowing-like motor program and its underlying neural basis had not been described. Thus, to understand the principles of the modular organization of the feeding circuitry, it was necessary to identify neurons that trigger swallowing-like activity. Toward 


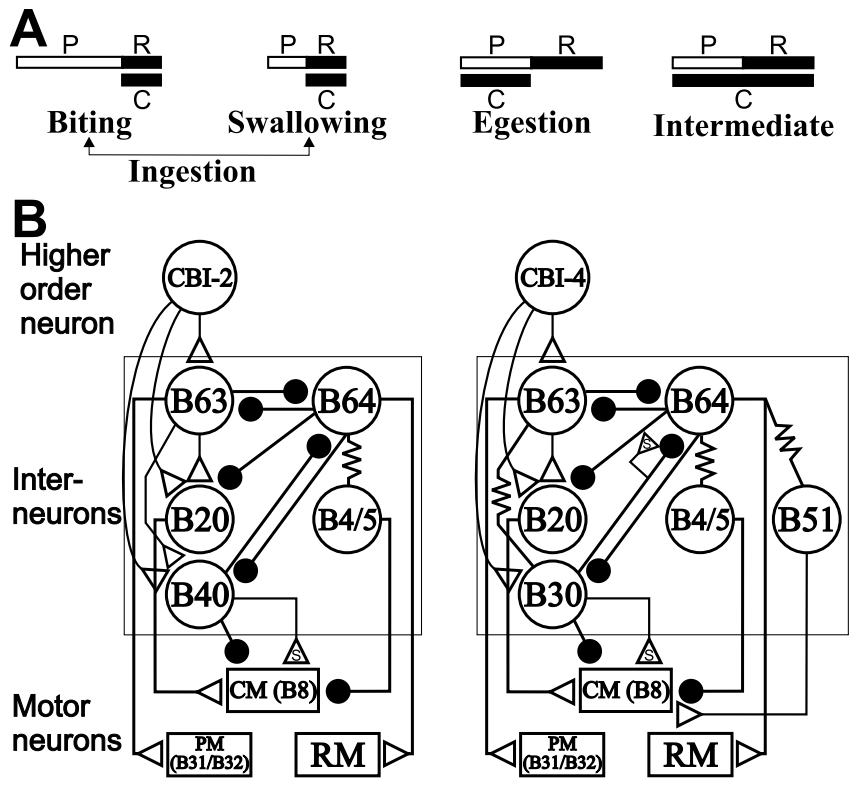

Figure 1. Multiple motor patterns that resemble feeding-related behavior in Aplysia and the networks that may underlie them. $A$, Classification of motor programs and their correspondence to behavior. Each cycle of a motor program, regardless of what type of motor program it is, comprises a protraction $(\mathrm{P})$ phase that is followed by a retraction $(\mathrm{R})$ phase. Motor programs can be classified into three basic forms on the basis of phasing of activity of radula closure $(C)$ motor neuron $\mathrm{B} 8$ relative to protraction-retraction. If closure activity occurs predominantly during retraction, the program is ingestive; if closure activity predominantly occurs during protraction, the program is egestive; and finally, if closure activity is moderate during both protraction and retraction, the program is intermediate. Furthermore, ingestive motor programs can be classified into two subtypes on the basis of duration of protraction. If the protraction is long, the motor program is biting-like; if the protraction is short, the program is swallowing-like. Biting-, swallowing-, and egestive-like programs likely correspond to three functionally effective forms of feeding behavior observed in intact animals. Intermediate programs have been observed when radula closure is monitored extracellularly in intact animals or intracellularly in isolated CNS (Morton and Chiel 1993a,b; Morgan et al. 2002), but the exact behavioral significance of this type of program remains to be determined. $B$, Schematic diagrams of the feeding circuits that produce multiple motor outputs. On the left, the circuit that is activated by $\mathrm{CBI}-2$ and mediates ingestive-biting-like, egestive, and intermediate programs is depicted and is based on previous work. On the right, the circuit that is activated by CBI-4 and mediates ingestiveswallowing-like, egestive, and intermediate programs is shown for comparison and is based primarily on work described in this paper. In both circuits, motor programs are initiated when CBI-2 or CBI-4 activates B63 to generate protraction. B63 and B64 reciprocally inhibit each other and mediate the alternating activity in protraction motor neurons (PM) (e.g., B31/32) and retraction motor neurons (RM). B64 is activated and the retraction phase is generated when polysynaptic excitation (data not shown) from protraction interneurons (e.g., CBI-2 and B63) overcomes inhibitory input to $\mathrm{B} 64$. The type of motor programs is determined by the phasing of activity of radula closure motor neurons (CM) (e.g., B8). In rejection-like programs (CBI-2 and $\mathrm{CBI}-4)$, $\mathrm{CM}$ activity during protraction is mediated by excitation that originates in $\mathrm{B} 20$ and inhibition that originates in $\mathrm{B} 4 / 5$. In biting-like ( $\mathrm{CBI}-2$ ) and swallowing-like ( $\mathrm{CBI}-4)$ programs, CM activity during retraction is mediated by fast inhibition and slow excitation that originates in $B 40$ and B30, respectively. Furthermore, the long protraction of biting-like programs is mediated by inhibition of $B 64$ by $B 40$, whereas the short protraction of swallowing-like programs is mediated by slow excitation of $\mathrm{B} 64$ by $\mathrm{B} 30$. In intermediate programs ( $\mathrm{CBI}-2$ and $\mathrm{CBI}-4$ ), $\mathrm{CM}$ activity in both protraction and retraction is mediated by coactivity of $\mathrm{B} 20$ and B40 or B30. Finally, B51 can be activated in some (BI-4-elicited programs to mediate hyper-retraction. Open triangle, Excitation; closed circle, inhibition; s, slow synaptic actions; zig-zagged line, electrical coupling.

this end, we turned our attention to CBI-4, a second CBI that is capable of driving robust motor programs (Rosen et al., 1991). The interneuronal circuit that is activated by stimulation of CBI- 4 is depicted in Fig. $1 B$ (right panel) and is characterized in previous work and in this study.

\section{Characteristics of motor programs elicited by CBI-4 and} cluster analysis

We identified the types of motor programs elicited by stimulation of CBI-4 using established criteria (Fig. $1 \mathrm{~A}$ ) (Morton and Chiel, 1993a,b; Morgan et al., 2002). We found that like CBI-2, CBI-4 can drive ingestive and egestive motor programs (Fig. $2 A$ ). Similar to motor programs elicited by other means, we observed that protraction (open bar) preceded retraction (filled bar). The radula closure motoneuron B8 could be predominantly active during either protraction or retraction. In Figure 2, A1 and A2, B8 activity occurred primarily during the retraction phase, indicating that these programs were ingestive. Conversely, in Figure 2, $A 3$ and $A 4, \mathrm{~B} 8$ activity occurred primarily during the protraction phase, indicating that these programs were egestive. The variability of CBI-4-evoked motor programs was mostly a result of different preparations and different stimulus conditions in the same preparation. In a given preparation when the same stimulation paradigm was used, buccal ganglia tended to produce similar motor programs.

In a subset of CBI-4-elicited programs, however, a second burst of B8 activity occurred during the late part of retraction (Fig. 2A2,A4, gray bar). Interestingly, protraction and retraction as well as closure activity in either ingestive (Fig. 2, compare A1, $A 2$ ) or egestive (Fig. 2, compare A3, A4) programs were qualitatively similar regardless of whether the second B8 burst occurred. During the retraction phase of ingestive programs with a second B8 burst, B8 activity (shown more clearly in instantaneous frequency plots of B8 in Fig. 2A2, top) quickly peaked in the first burst after the initiation of retraction and then slowly declined, similar to programs without a second B8 burst (Fig. 2A1). During the early part of the retraction phase of egestive motor programs with a second B8 burst, B8 activity (shown more clearly in instantaneous frequency plots of B8 in Fig. 2A4, top) was weak, similar to egestive programs without a second B8 burst (Fig. 2A3). This suggests that the second $\mathrm{B} 8$ burst may represent a phase that is added onto retraction. On the basis of this observation and two sets of quantitative data provided below, we defined the phase during which the second B8 burst occurs as hyper-retraction, following a previous convention (Evans and Cropper, 1998). The second B8 burst has also been observed in egestive motor programs recorded from intact animals (Morton and Chiel, 1993a), during which the activity of the radula closure motor neuron B8 was monitored via a recording from the RN. The same study did not report a second B8 burst for ingestive motor programs, which could well be attributable to the fact that activity in the RN was too high throughout retraction and hyper-retraction, and thus it was difficult to distinguish the early B8 burst from the second B8 burst. In fact, in our recording of CBI-4-elicited ingestive motor programs (Fig. 2A2), it was also difficult to distinguish the two B8 bursts in the RN recording, but they were obvious in intracellular recordings of $\mathrm{B} 8$. The separation of the two B8 bursts can be clearly seen as a drop of the instantaneous B8 firing frequency during retraction (Fig. 2A2, top). The second B8 burst-hyperretraction in CBI-4 programs occurred regardless of whether motor programs were elicited in single cycles through brief stimulation of CBI-4 or multiple cycles through prolonged stimulation of CBI-4. In programs with multiple cycles, hyper-retraction often occurred repetitively, i.e., it was not limited to the last cycle.

For a more quantitative description of CBI-4-elicited programs, we performed two analyses: classification of programs into different types and comparison of multiple parameters for programs with or without a second B8 burst. For motor program classification, we plotted B8 activity during protraction and re- 

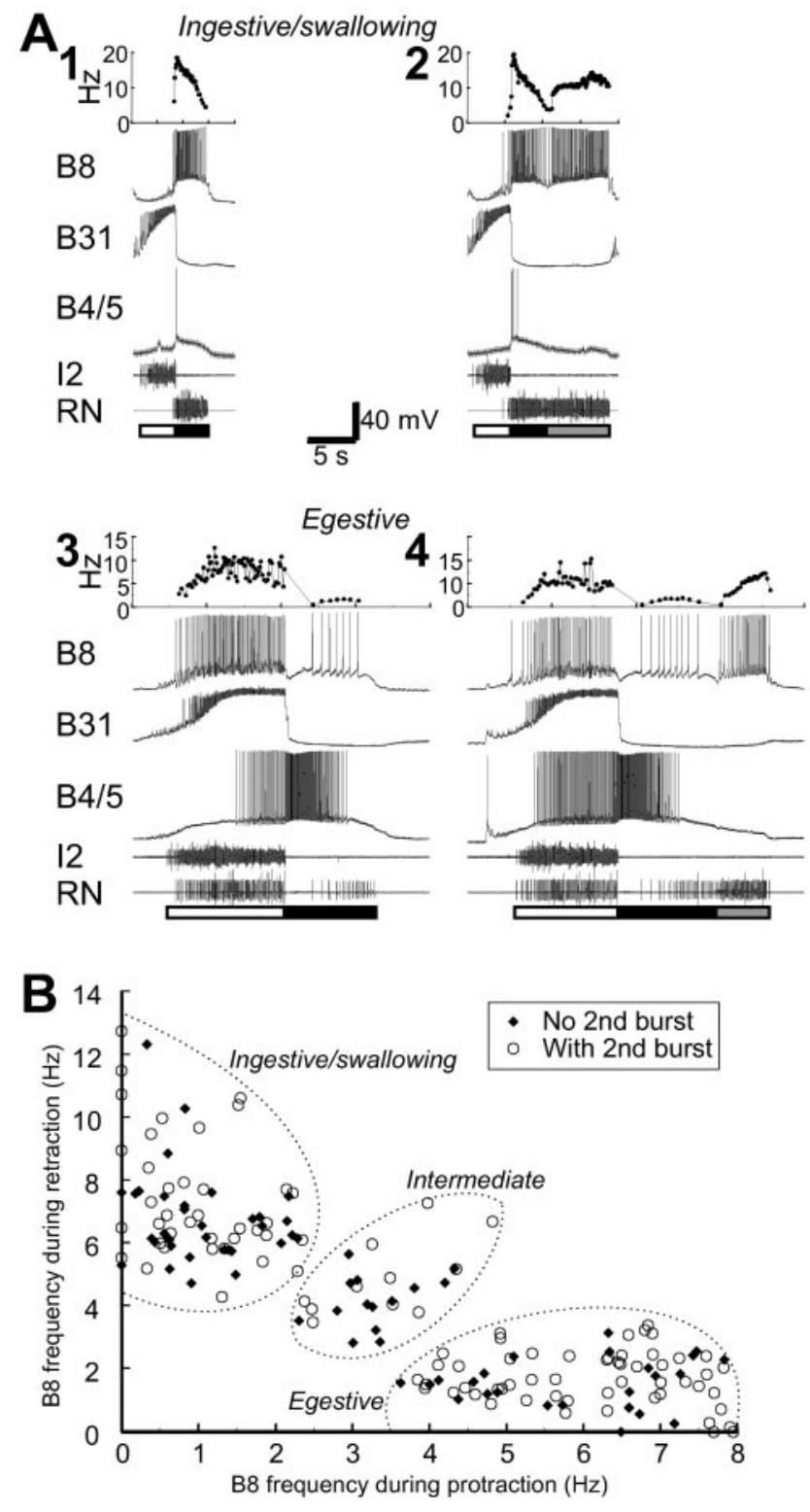

Figure 2. Characterization of motor programs elicited by CBI-4 stimulation. $A$, Examples of programs elicited by $\mathrm{CBI}-4$. Protraction (open bar) is monitored by activity in the $\mathrm{I} 2$ nerve and B31, retraction (filled bar) by depolarization of B4/5 after the termination of $\mathrm{I} 2$ activity, hyperpolarization in B31, and closure activity in the RN and B8. Top, Plots of instantaneous frequency of $B 8$ during program. The motor programs in $A 1$ and $A 2$ were ingestive, because $B 8$ was active primarily during retraction. The motor programs in $A 3$ and $A 4$ were egestive, because $B 8$ was active primarily during protraction. $A 2$ and $A 4$ were similar to $A 1$ and $A 3$, respectively, except that there was a second $B 8$ burst (gray bar) observed during the late part of retraction (hyperretraction). Thus, motor programs in $A 2$ or $A 4$ are not considered as different types of programs from those in $A 1$ or $A 3$, but two different variants of the same type of program. $B$, Cluster analysis. Plot of average $B 8$ frequency during protraction versus that during retraction (excluding the second B8 burst-hyper-retraction) for 175 (BI-4-elicited motor programs is shown. Three clusters enclosed by dotted lines are based on visual inspection and produced the smallest determinant of within-group covariance matrix, suggesting that the data can be separated into three clusters: ingestive, intermediate, and egestive. The distribution of data points for motor programs with or without a second B8 burst is mixed, suggesting that the occurrence of the second $B 8$ burst is independent of $B 8$ activity during protraction and early retraction. Resting membrane potentials (in $\mathrm{mV}$ ): $\mathrm{B} 8,-57 ; \mathrm{B} 31,-65 ; \mathrm{B} 4 / 5,-66$.

traction in $175 \mathrm{CBI}-4$-elicited motor programs in 26 preparations. For this purpose, we did not count B8 activity during hyper-retraction. This methodology follows that of Morton and Chiel (1993a), who excluded the second B8 burst when consid- ering whether motor programs were ingestive or egestive, because behavioral consequences of B8 activity appeared to be manifested during protraction and early retraction. Data are shown in Figure $2 B$, in which each point represents one cycle, and cycles with or without a second B8 burst are shown in different symbols. The two types of symbols had a similar distribution and were intermixed, indicating that the occurrence of the second B8 burst is independent of $\mathrm{B} 8$ activity during protraction and early retraction. Therefore, we used the pooled data to perform a cluster analysis (Morgan et al., 2002).

For cluster analysis, we tested four different strategies for partitioning data. First, we separated data into three groups on the basis of visual inspection (Fig. $2 \mathrm{~B}$, groups enclosed with dotted lines). Second, we separated data into two groups, an upper-left group and a lower-right group. The upper-left group consisted of the ingestive group and half of the intermediate group in Figure $2 \mathrm{~B}$. The lower-right group consisted of the egestive group and the other half of the intermediate group in Figure $2 B$. Third, we treated data as one group. Finally, we separated the data into three groups by random assignment of the data points. We performed two such random assignments.

For each partition, we calculated the within-group covariance matrix for one group or pooled within-group covariance matrices for multiple groups. We then took the determinant of withingroup covariance matrix as $|\mathrm{W}|$, with the smallest $|\mathrm{W}|$ indicating the best partitioning strategy (Marriott, 1971; Krzanowski and Lai, 1988). The first partition (three groups) resulted in the smallest $|\mathrm{W}|$ (2.04), whereas the $|\mathrm{W}|$ for the second partition (two groups) was 4.35 and $|\mathrm{W}|$ for the third partition (one group) was 18.25. The $|\mathrm{W}|$ values for the two random partitions were 18.35 and 18.33, which were similar to the $|\mathrm{W}|$ for the one-group partition. This suggests that the separation of data into three clusters on the basis of visual observation may be the best strategy for partitioning data. As in Figure $2 B$, we classified the upper-left cluster with $\mathrm{B} 8$ mostly active during retraction as ingestive, the lower-right cluster with B8 mostly active during protraction as egestive, and the middle cluster with B8 similarly active during protraction and retraction as intermediate. It is not clear whether these intermediate programs will generate effective behaviors, but intermediate programs were observed originally in intact animals (Morton and Chiel, 1993a); later, they were also observed when activity was triggered by CBI-2 stimulation in the isolated CNS (Morgan et al., 2002).

After classification of CBI-4-elicited activity, we determined that $53 \%$ of the ingestive motor programs, $42 \%$ of the intermediate motor programs, and $63 \%$ of the egestive motor programs had a second B8 burst. For a more quantitative comparison of CBI-4 programs with or without a second B8 burst, we measured the duration of B8 activity and B8 firing frequency during protraction, retraction, and hyper-retraction. Average data are shown in Figure $3 A$. These data indicate that regardless of whether a second B8 burst was absent or present, the duration of both protraction and retraction was similar in ingestive, egestive, or intermediate programs. In addition, regardless of whether a second B8 burst was absent or present, B8 firing frequency during protraction and retraction was similar in ingestive, egestive, or intermediate programs. Thus these data validate the pooling of programs with or without a second B8 burst in cluster analysis and further support the notion that the second B8 burst (hyperretraction) is a separate phase. For these reasons, we considered CBI-4-elicited ingestive motor programs with or without hyperretraction not as different types of program but different variants of the same behavior. The same statement applies to CBI-4- 


\section{A Protraction}
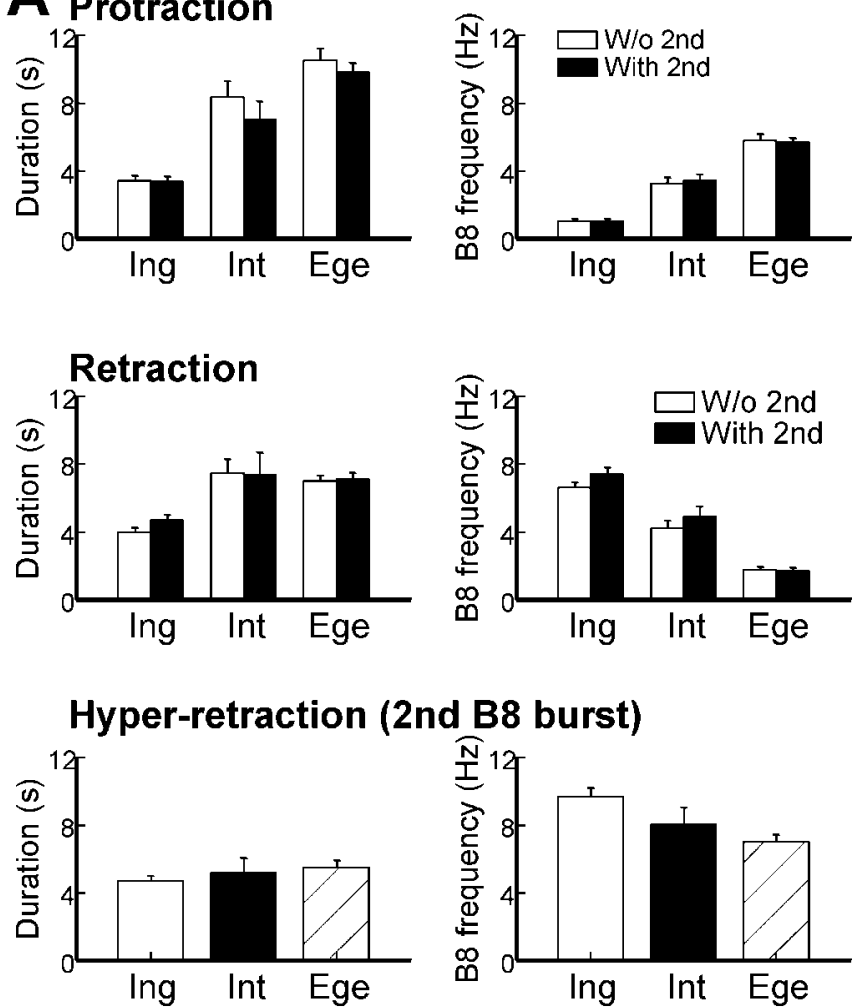

B

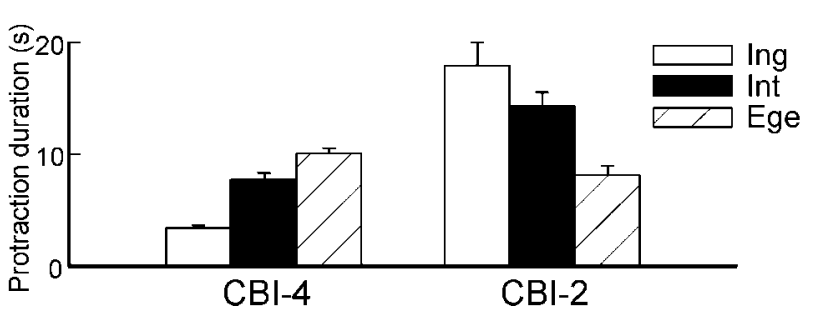

Figure 3. Parametric features of $C B I-4$-elicited motor programs. $A$, Comparison of $C B I-4-$ elicited programs with or without a second B8 burst. Regardless of whether a second B8 burst was absent or present, the duration of both protraction and retraction (excluding hyperretraction) was similar in ingestive (protraction: $3.4 \pm 0.3 \mathrm{sec}, n=29$, vs $3.4 \pm 0.2 \mathrm{sec}, n=$ 35 , unpaired two-tailed t test, $t=0.7, p>0.05$; retraction: $4.0 \pm 0.2 \mathrm{sec}, n=29$, vs $4.7 \pm 0.3$ sec, $n=35$, unpaired two-tailed $t$ test, $t=0.6, p>0.05$ ), egestive (protraction: $10.5 \pm 0.7$ $\mathrm{sec}, n=19$, vs $9.8 \pm 0.5 \mathrm{sec}, n=34$, unpaired two-tailed $t$ test, $t=1.0, p>0.05$; retraction, $7.0 \pm 0.3 \mathrm{sec}, n=19$, vs $7.1 \pm 0.3 \mathrm{sec}, n=34$, unpaired two-tailed $t$ test, $t=0.1, p>0.05$ ), or intermediate (protraction: $8.4 \pm 0.9 \mathrm{sec}, n=13$, vs $7.0 \pm 1.0 \mathrm{sec}, n=12$, unpaired two-tailed $t$ test, $t=1.2, p>0.05$; retraction: $7.5 \pm 0.8 \mathrm{sec}, n=13$, vs $7.4 \pm 1.3 \mathrm{sec}, n=12$, unpaired two-tailed $t$ test, $t=0.02, p>0.05$ ) programs. In addition, regardless of whether a second $B 8$ burst was absent or present, $B 8$ firing frequency during protraction and retraction was similar in ingestive (protraction: $1.03 \pm 0.1 \mathrm{~Hz}, n=29$, vs $1.04 \pm 0.1 \mathrm{~Hz}, n=35$, unpaired two-tailed $t$ test, $t=0.4, p>0.05$; retraction: $6.6 \pm 0.3 \mathrm{~Hz}, n=29$, vs $7.4 \pm 0.3 \mathrm{~Hz}, n=35$, unpaired two-tailed $t$ test, $t=1.7, p>0.05$ ), egestive (protraction: $5.8 \pm 0.3 \mathrm{~Hz}, n=19$, vs $5.7 \pm 0.2 \mathrm{~Hz}, n=34$, unpaired two-tailed $t$ test, $t=0.4, p>0.05$; retraction: $1.8 \pm 0.15 \mathrm{~Hz}$, $n=19$, vs $1.7 \pm 0.15 \mathrm{~Hz}, n=34$, unpaired two-tailed $t$ test, $t=0.03, p>0.05$ ), or intermediate (protraction: $3.3 \pm 0.3 \mathrm{~Hz}, n=13$, vs $3.4 \pm 0.4 \mathrm{~Hz}, n=12$, unpaired two-tailed $t$ test, $t=0.5, p>0.05$; retraction: $4.2 \pm 0.4 \mathrm{~Hz}, n=13, \mathrm{vs} 4.9 \pm 0.5 \mathrm{~Hz}, n=12$, unpaired two-tailed $t$ test, $t=1.8, p>0.05)$ programs. The duration of hyper-retraction was similar during ingestive ( $4.7 \pm 0.2 \mathrm{sec} ; n=35)$, intermediate $(5.2 \pm 0.8 \mathrm{sec} ; n=12)$, or egestive $(5.5 \pm 0.4 \mathrm{sec} ; n=34)$ motor programs (one-way ANOVA; $\left.F_{(2,78)}=1.4 ; p>0.05\right)$. In contrast, $B 8$ firing frequency was higher $(9.7 \pm 0.5 \mathrm{~Hz} ; n=35)$ in ingestive programs, lower $(8.1 \pm 0.9$ $\mathrm{Hz} ; n=12)$ in intermediate programs, and lowest $(7.0 \pm 0.4 \mathrm{~Hz} ; n=34)$ in egestive programs [one-way ANOVA; $F_{(2,78)}=7.9 ; p<0.001 ;$ a Bonferroni multiple comparisons test of the three different group pairs showed significant difference between $B 8$ firing frequency in ingestive programs and that in egestive programs ( $t=4.0 ; p<0.001)$, no significant difference between that in ingestive programs and that in intermediate programs $(t=1.6 ; p>0.05)$, and elicited egestive and intermediate motor program with or without hyper-retraction.

Data (Fig. $3 A$ ) also indicate that the duration of hyperretraction was similar during ingestive, intermediate, or egestive motor programs. In contrast, B8 firing frequency was higher in ingestive programs, lower in intermediate programs, and lowest in egestive programs.

\section{CBI-4-elicited ingestive programs are swallowing-like}

The analyses described above allowed us to compare motor programs elicited by CBI-2 and CBI-4. If one compares CBI-2- and CBI-4-elicited programs in terms of the phasing of B8 activity during protraction and retraction, $\mathrm{CBI}-2$ - and $\mathrm{CBI}-4$-elicited programs are similar because programs elicited by either CBI can be classified as ingestive, intermediate, or egestive. CBI-4-elicited motor programs, however, had two major characteristics that distinguished them from CBI-2-elicited motor programs.

First, the protraction duration in CBI-4-elicited ingestiveswallowing-like motor programs was short (Figs. 2A, 3B) (examples of CBI-2-elicited motor programs are shown in Morgan et al., 2002, their Fig. 1). Because we have shown that several parameters, including protraction duration, are not determined by whether CBI-4-elicited motor programs have a second B8 burst, we pooled all CBI-4 data to obtain the protraction duration measure. Protraction is the shortest in CBI-4-elicited ingestive programs and the longest in $\mathrm{CBI}-4$-elicited egestive programs, whereas it is the opposite for CBI-2-elicited programs. Previously, we classified CBI-2-elicited ingestive motor programs as biting-like, in part because they have a long protraction duration (Jing et al., 2003). These data therefore suggest that CBI-4elicited ingestive programs are swallowing-like because they have a short protraction duration (Hurwitz et al., 1996).

Second, CBI-4-elicited motor programs differ from CBI-2elicited motor programs in that there is a second B8 burst in about half of the CBI-4-elicited programs (Fig. 2A2,A4), whereas a second $\mathrm{B} 8$ burst is rarely observed in CBI-2-elicited motor programs. The second $\mathrm{B} 8$ burst in CBI-4-elicited ingestiveswallowing-like programs would presumably serve to pull food deeper into the esophagus when food was successfully grasped by the radula, consistent with a swallowing movement (Weiss et al., 1986). The occurrence of the second $B 8$ burst in about half of the CBI-4-elicited swallowing-like programs would make the duration of total retraction (including the early phase of retraction and hyper-retraction), on average, longer than the retraction phase in CBI-2-elicited biting-like programs. This is consistent

between that in intermediate programs and that in egestive programs $(t=1.1 ; p>0.05)] . B$ Comparison of protraction duration in $\mathrm{CBI}-4$ - and $\mathrm{CBI}-2$-elicited programs. Data for CBI-4elicited programs with or without a second B8 burst were pooled. In CBI-4-elicited motor programs, the average protraction duration was shortest $(3.4 \pm 0.2 \mathrm{sec} ; n=64)$ when programs were ingestive, longer $(7.7 \pm 0.5 \mathrm{sec} ; n=25)$ when programs were intermediate, and longest $(10.1 \pm 0.4 \mathrm{sec} ; n=53)$ when programs were egestive [one-way ANOVA; $F_{(2,144)}=124.1$; $p<0.0001 ;$ a Bonferroni multiple comparisons test of the three different group pairs showed significant difference among all group pairs $(p<0.001)$ ]. In contrast, the reverse was true for (BI-2-elicited motor programs. The average protraction duration was longest (17.9 $\pm 2.1 \mathrm{sec}$; $n=19)$ when programs were ingestive, shorter $(14.3 \pm 1.2 \mathrm{sec} ; n=4)$ when programs were intermediate, and shortest $(8.2 \pm 0.8 \mathrm{sec} ; n=19)$ when programs were egestive [one-way ANOVA; $F_{(2,39)}=37.7 ; p<0.0001$; a Bonferroni multiple comparisons test of the three different group pairs showed significant difference between protraction duration in ingestive programs and that in egestive programs $(t=8.6 ; p<0.001)$ and between that in intermediate programs and that in egestive programs $(t=3.1 ; p<0.05)$, but no significant difference between that in ingestive programs and that in intermediate programs $(t=1.9 ; p>0.05)]$. Ing, Ingestive; Int, intermediate; Ege, egestive. Error bars represent SEM. 

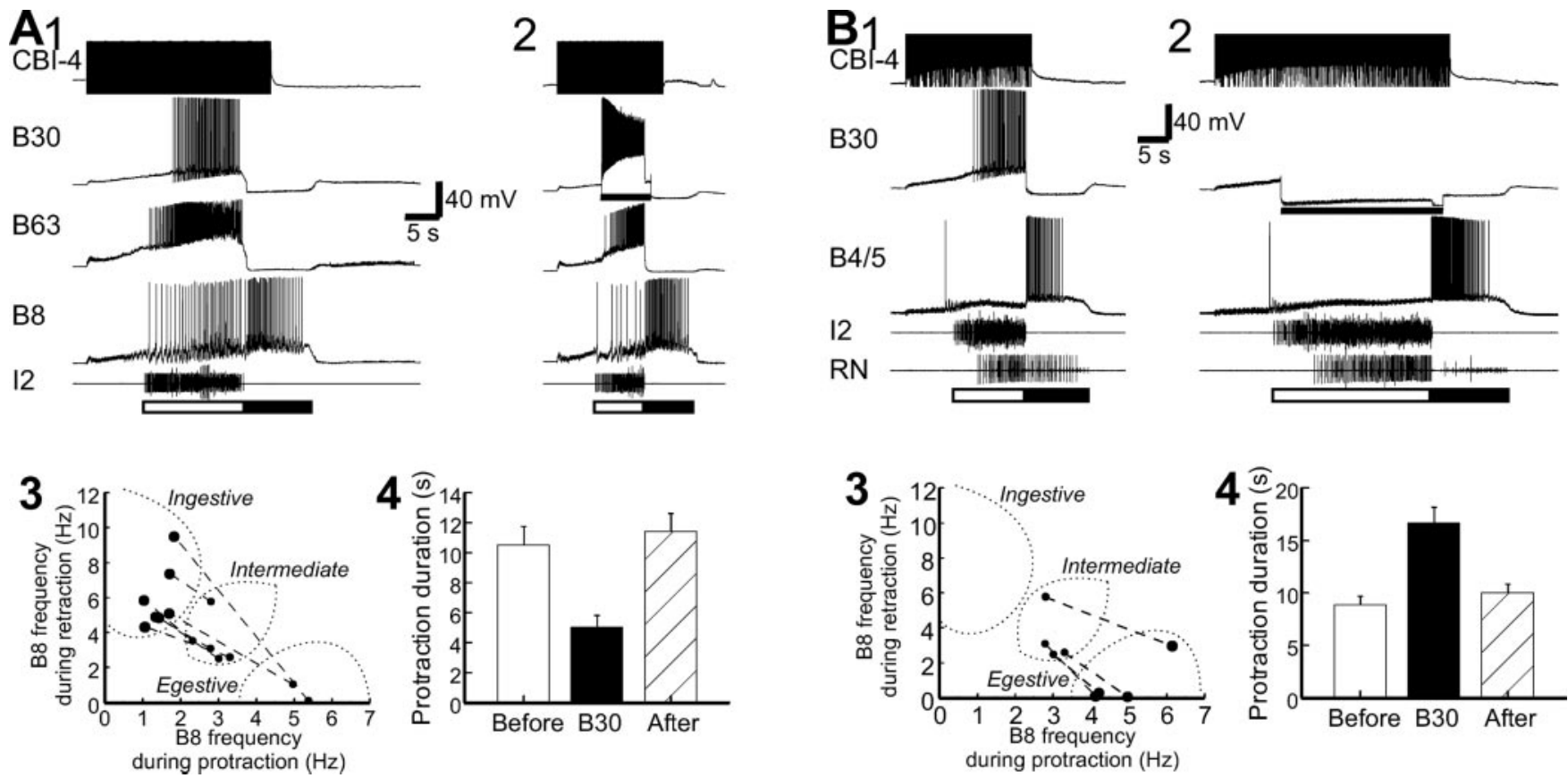

Figure 4. B30 activity enhances the ingestiveness of $\mathrm{CBI}-4$-elicited programs and shortens protraction duration. $A, \mathrm{CBI}-4$ stimulation induced moderate activity in $\mathrm{B} 30$, and the program was intermediate because $B 8$ was active during both protraction and retraction (A1). Activation of $B 30$ through depolarization (bar) made the $(B \mid-4$-elicited program more ingestive and shortened protraction (A2). $A 3$, $A 4$, Group data $(n=8)$ for the effect of B30 depolarization on B8 activity and protraction duration [repeated-measures ANOVA; $F_{(2,12)}=44.5 ; p<0.0001 ; \mathrm{a}$ Bonferroni multiple comparisons test of different group pairs showed that protraction duration during B30 depolarization was significantly different from the "before" $(t=7.5 ; p<0.001)$ and "after" $(t=8.7 ; p<$ $0.001)$ groups, whereas protraction duration of the before group was not significantly different from the duration of the after group $(t=1.2 ; p>0.05)]$. B, In another preparation, $(B \mid-4$ stimulation induced moderate activity in B30, and the program was intermediate because radula closure in B8 (as monitored by activity in RN) was active during both protraction and retraction (B1). Hyperpolarization of $\mathrm{B} 30$ (bar) made the $\mathrm{CB}-4$-elicited program more egestive and lengthened protraction (B2). $B 3, B 4$, Group data $(n=4)$ for the effect of B30 hyperpolarization on $B 8$ activity and protraction duration [repeated-measures ANOVA; $F_{(2,6)}=88.5 ; p<0.0001$; a Bonferroni multiple comparisons test of different group pairs showed that protraction duration during B30 hyperpolarization was significantly different from the before $(t=12.3 ; p<0.001)$ and after $(t=10.5 ; p<0.001)$ groups, whereas protraction duration of the before group was not significantly different from the duration of the after group $(t=1.8 ; p>0.05)]$. In $A 3$ and $B 3$, data points with small symbols represent data obtained in control conditions and those with large symbols obtained during B30 stimulation (A3) or hyperpolarization (B3). Resting membrane potentials (in mV): $A$ : $(B \mid-4,-64 ; B 30,-57 ; B 63,-58 ; B 8,-65 ; B$ : $C B I-4,-61 ; B 30,-55 ; B 4 / 5,-69$. Error bars represent SEM.

with previous findings in intact animals (Cropper et al., 1990; Morton and Chiel, 1993a) that showed that swallowing had a somewhat longer retraction duration than biting. In contrast, it is not clear at present what role the second B8 burst may serve in egestive motor programs. In a previous study with intact animals (Morton and Chiel, 1993a), it was found that those egestive programs with a second B8 burst often occurred during transitions between ingestive and egestive motor programs, and vice versa.

\section{Role of the buccal interneuron $\mathrm{B} 30$ in swallowing-like motor programs}

The finding that $\mathrm{B} 8$ displays the same phasing characteristics in CBI-2- and CBI-4-elicited ingestive motor programs but that the two programs differ in the duration of protraction allowed us to examine whether such combinations of program features are implemented by behavior-independent modules, e.g., one module that encodes B8 phasing and another protraction duration, or by behavior-specific modules, each of which encodes both B8 phasing and protraction duration.

Our previous work (Jing and Weiss, 2002) demonstrated that B8 phasing in CBI-2-elicited ingestive-biting-like programs is mediated primarily by B40 interneurons (Fig. $1 \mathrm{~B}$ ). If each parameter is controlled by a common module in different behaviors, one would expect that B8 phasing in CBI-4-elicited ingestiveswallowing-like motor programs would also be mediated by $\mathrm{B} 40$. We found, however, that B40 generally is not active or is only weakly active in CBI-4-elicited programs, regardless of what types of programs they were (swallowing-like: $1.2 \pm 0.4 \mathrm{~Hz}, n=1$; intermediate: $0.7 \pm 0.6 \mathrm{~Hz}, n=8$; egestive: $1.1 \pm 0.4 \mathrm{~Hz}, n=20$; one-way ANOVA showed no significant difference among these data groups; $\left.F_{(2,44)}=0.3 ; p>0.05\right)$. In contrast, $B 40$ is strongly active $(9.9 \mathrm{~Hz})$ in CBI-2-elicited ingestive-biting-like programs (Jing and Weiss, 2002). The data suggest that $B 8$ phasing as well as the shorter protraction in CBI-4-elicited swallowing-like programs is mediated by a different interneuron(s).

We identified a novel interneuron in the buccal ganglion, which we named B30, that is active in swallowing-like motor programs elicited by CBI- 4 . There are two B30 interneurons in each hemiganglion; thus there are total of four B30 interneurons in the buccal ganglion. The B30 interneurons are located close to two previously identified cells, B34 (Hurwitz et al., 1997) and B40 (Jing et al., 2003), and typically are covered by superficial cells. To distinguish the two B30 interneurons, we have designated as $\mathrm{B} 30 \mathrm{~A}$ the one that is closer to $\mathrm{B} 34$, and we have designated as $\mathrm{B} 30 \mathrm{~B}$ the one that is closer to B40. The B30 neurons send their axons medially to cross the buccal commissure to the contralateral hemiganglion. Their axons do not exit the buccal ganglion and so B30 appears to be a local interneuron.

Like B40 and B20 (Jing and Weiss, 2001, 2002), B30 is active during the protraction phase of motor programs (Figs. $1 B, 4$ ). Consistent with a potential role of B30 in CBI-4-elicited swallowing-like programs, B30 is strongly active $(12.4 \pm 0.4 \mathrm{~Hz}$; $n=30)$ in swallowing-like programs, less active $(4.9 \pm 0.2 \mathrm{~Hz}$; $n=10)$ in intermediate programs, and weakly or not active 

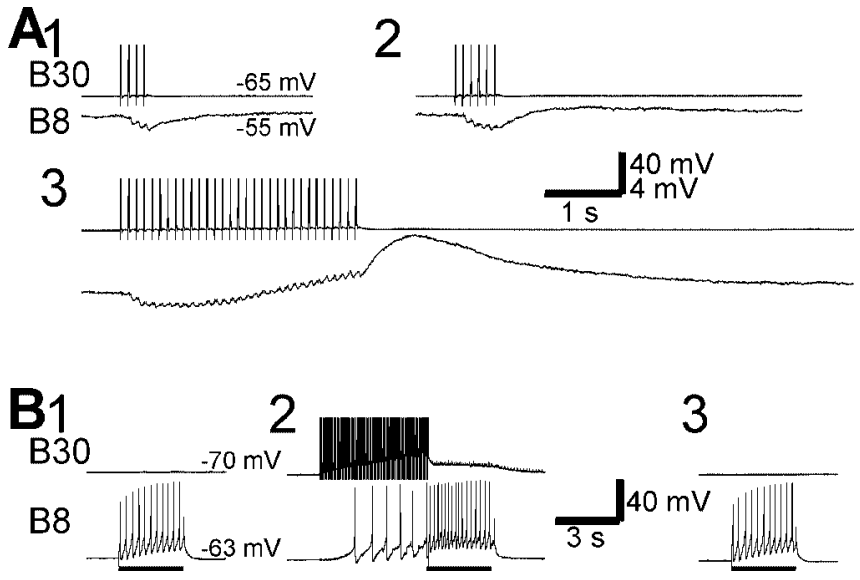

Figure 5. B30 produces fast IPSPs and a slow EPSP in B8. A, Stimulation of B30 with brief current pulses at $10 \mathrm{~Hz}$ for different durations elicited fast IPSPs and a slow EPSP. The slow EPSP was absent (A1) or small ( $A 2$ ) when $B 30$ was stimulated for brief periods but became obvious when $B 30$ was stimulated for $3 \sec (A 3)$. Recordings were made in high-divalent saline. $B$, Stimulation of $B 30$ at $20 \mathrm{~Hz}$ for 5 sec increased the $B 8$ firing frequency when the current pulse in B8 (bar) was injected after B30 activity (from left to right: 12, 16, and 12 spikes in B8). For group data $(n=4)$, the number of spikes in B8 was as follows: before B30, 13.3 \pm 1.1 ; with B30, $18.3 \pm 1.1$; after B30, $13.5 \pm 1.0$ [repeated-measures ANOVA; $F_{(2,6)}=32.7 ; p<0.001 ; \mathrm{a}$ Bonferroni multiple comparisons test of different group pairs showed that the number of $B 8$ spikes with B30 activation was significantly different from that in the before $(t=7.2 ; p<0.01)$ and the after $(t=6.8 ; p<0.01)$ groups, whereas the number of $B 8$ spikes in the before group was not significantly different from that in the after group $(t=0.4 ; p>0.05)]$. Recordings were made in normal saline. Voltages to the right of the records of individual neurons indicate membrane potentials.

$(3.1 \pm 0.6 \mathrm{~Hz} ; n=14)$ in egestive programs [one-way ANOVA; $F_{(2,43)}=53.8 ; p<0.0001$; a Bonferroni multiple comparisons test of different group pairs showed that B30 activity in swallowinglike programs was significantly different from that in intermediate $(t=5.9 ; p<0.001)$ and egestive programs $(t=9.6 ; p<$ $0.001)$, whereas B30 activity in intermediate programs was not significantly different from that in egestive programs $(t=1.7 ; p>$ $0.05)]$.

The functional role of B30 in CBI-4-elicited motor programs was determined by depolarization or hyperpolarization experiments (Fig. 4). In Figure 4A, B30 was moderately active in CBI4-elicited intermediate programs, whereas B8 was active during both protraction and retraction. Stronger activation of B30 through current injection had two effects on programs: they became more ingestive (less B8 activity during protraction and more during retraction), and protraction duration became shorter (Fig. 4A2) $(n=8)$. Conversely, hyperpolarization of B30 made programs more egestive (more $\mathrm{B} 8$ activity during protraction and less activity during retraction) and increased protraction duration (Fig. 4B2) $(n=4)$. Group data are shown in Figure 4, A3 and $A 4$ and $B 3$ and $B 4$, respectively, and statistical analyses are shown in the Figure 4 legend. Thus, B30 appears to mediate both B8 phasing and short protraction duration for CBI-4-elicited swallowing-like programs.

To determine how B30 may promote B8 activity during retraction and inhibit activity during protraction, we examined synaptic connections from B30 to B8. We found that B30 exerted fast inhibition and slow excitation in B8 (Fig. $5 A)(n=14)$. To determine whether the slow EPSPs were functional, we tested the effects of B30 activity on the excitability of B8. Activation of B30 at $20 \mathrm{~Hz}$ just before $3 \mathrm{sec}$ current pulses were injected into B8 increased B8 firing frequency (Fig. $5 B)(n=4)$, which suggests that the slow EPSP is functional.
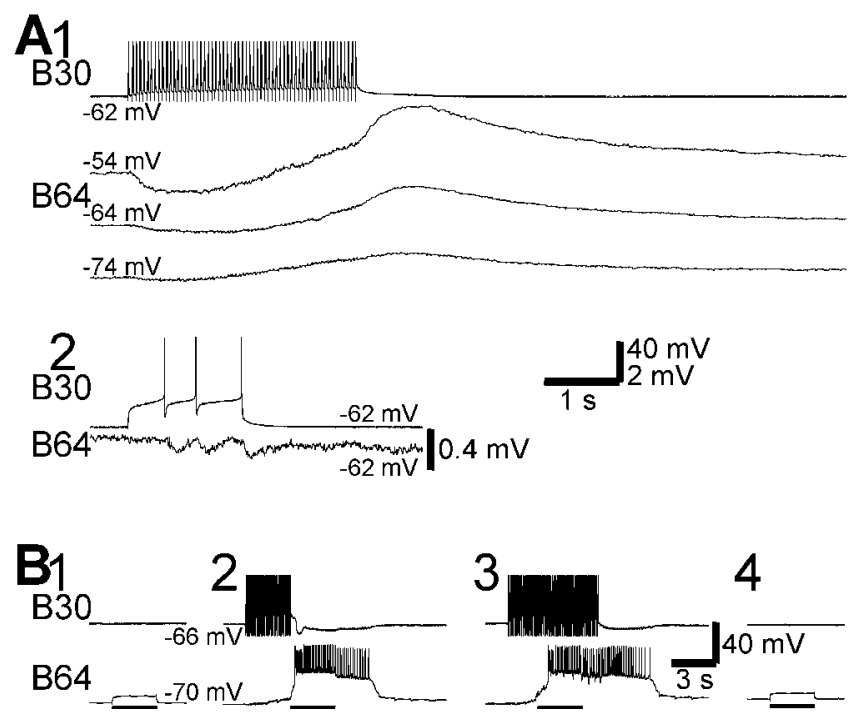

Figure 6. B30 elicited slow EPSPs in retraction-interneuron B64. A, Monosynaptic connections from $B 30$ to $B 64$. $A 1$, The slow EPSPs in $B 64$ elicited by $B 30$ were more prominent when $B 64$ was more depolarized. At a low firing frequency of B30, only small, fast IPSPs that followed B30 spikes one-for-one were obvious (A2). The slow EPSPs became obvious when B30 was stimulated with a train of spikes at a faster rate $(A 1)$. Recordings were made in high-divalent saline. $B, B 30$ activity enhanced B64 excitability and plateau generation. Without B30 activity (B1, B4), a 3 sec subthreshold current pulse in B64 (bar) did not induce spiking in B64. When B30 was stimulated at $20 \mathrm{~Hz}$ for $3 \mathrm{sec}$ immediately preceding the current pulse in B64, B64 fired a burst of spikes and generated a plateau potential that outlasted the current pulse injected in B64 (B2). When B30 was stimulated at $20 \mathrm{~Hz}, 3$ sec before and throughout the current pulse in B64, B64 generated a plateau potential with a brief delay, and the plateau outlasted the current pulse injected in B64 (B3). Recordings were made in normal saline. Voltages to the left or right of the records of individual neurons indicate membrane potentials.

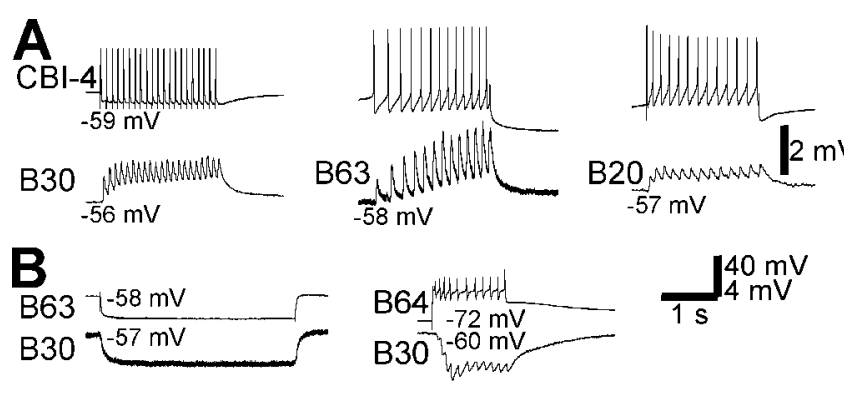

Figure 7. Synaptic connectivity of $\mathrm{CBI}-4$ and $\mathrm{B} 30$. A, Stimulation of $\mathrm{CBI}-4$ elicited fast monosynaptic EPSPs in B30, B63, and B20 that followed presynaptic spikes one-for-one. Recording was made in high-divalent saline. $B, B 30$ may be electrically coupled to B63 because current injection in B63 induced hyperpolarizing response in B30 (normal saline). B64 elicited fast monosynaptic IPSPs in B30 that followed presynaptic spikes one-for-one (high-divalent saline). Voltages to the left of the records of individual neurons indicate membrane potentials.

To determine how $\mathrm{B} 30$ may shorten protraction, we examined $\mathrm{B} 30$ actions on the retraction-phase neuron B64. We found that B30 elicited fast IPSPs and slow EPSPs in B64 (Fig. 6A) $(n=7)$. Fast IPSPs were typically small when B64 was held near resting potential but were more obvious in high-divalent saline (Fig. $6 A 2$ ). Slow EPSPs became larger when B64 was depolarized (Fig. $6 \mathrm{A1}$ ) and required $\mathrm{B} 30$ activity with multiple spikes at $>5 \mathrm{~Hz}$. We also tested the effect of B30 stimulation on B64 excitability (Fig. 6B) $(n=4)$. Subthreshold current pulses were applied to B64 every $30 \mathrm{sec}$. When B30 was stimulated just before or during current pulses in B64, B64 generated a plateau potential after B30 stimulation and fired spikes throughout the plateau. These data suggest that slow EPSPs from B30 to B64 are indeed functional 

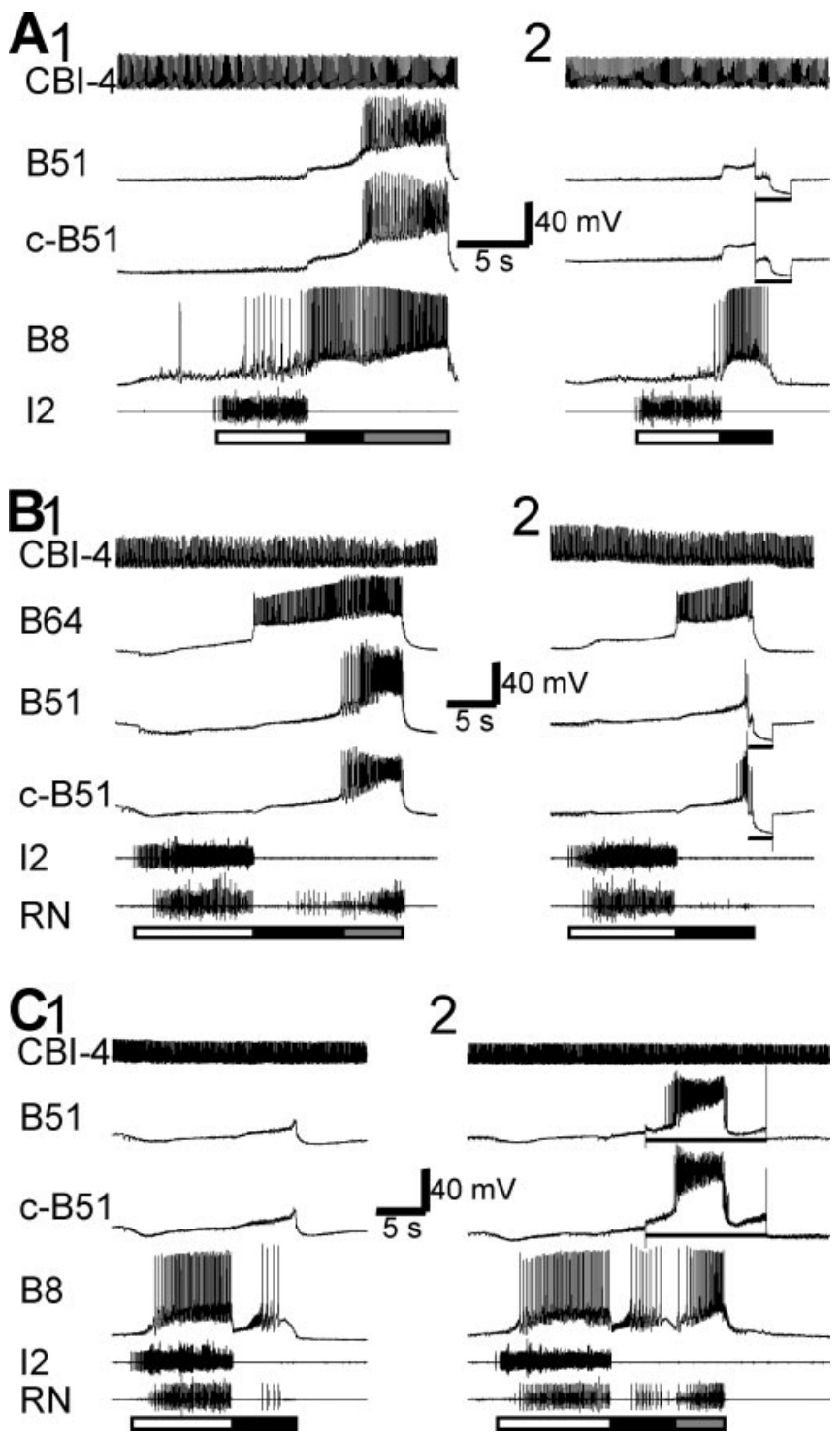

Figure 8. B51 is both necessary and sufficient for the second B8 burst in CBI-4-elicited motor programs. $A, \mathrm{CBI}-4$ stimulation elicited an ingestive program with a second $\mathrm{B} 8$ burst (gray bar) (A1). B51 hyperpolarization (bar) prevented the occurrence of the second B8 burst (A2). Importantly, in these ingestive programs $(n=3)$, B8 firing frequency during protraction (1.1 $\pm 0.2 \mathrm{vs}$ $0.8 \pm 0.1 \mathrm{~Hz}$; paired two-tailed $t$ test; $t=1.1 ; p>0.05)$ or during early retraction $(9.4 \pm 0.2$ vs $9.3 \pm 0.2 \mathrm{~Hz}$; paired two-tailed $t$ test; $t=0.2 ; p>0.05$ ) was not influenced by B51 hyperpolarization. $B,(\mathrm{Bl}-4$ stimulation elicited an egestive program with a second $\mathrm{B} 8$ burst (gray bar; as monitored by activity in RN) (B1). B51 hyperpolarization (bar) prevented the second B8 burst from occurring (B2). Similarly, in these egestive programs $(n=4)$, B8 firing frequency during protraction ( $5.5 \pm 0.7$ vs $5.7 \pm 0.8 \mathrm{~Hz}$; paired two-tailed $t$ test; $t=1.6 ; p>$ $0.05)$ or during early retraction $(1.4 \pm 0.4 \mathrm{vs} 1.2 \pm 0.5 \mathrm{~Hz}$; paired two-tailed $t$ test; $t=0.9 ; p>$ 0.05 ) was not influenced by B51 hyperpolarization. C, An egestive motor program without a second $B 8$ burst was induced by CBI-4 stimulation (C1). Injection of a depolarizing current into B51 (bar) induced B51 firing and a second burst of B8 firing (gray bar) at the end of retraction (C2). Similarly, in these programs $(n=3)$, B8 firing frequency during protraction ( $6.3 \pm 0.6 \mathrm{vs}$ $6.1 \pm 0.5 \mathrm{~Hz}$; paired two-tailed $t$ test; $t=0.9 ; p>0.05)$ or during early retraction $(1.2 \pm 0.3$ vs $1.4 \pm 0.3 \mathrm{~Hz}$; paired two-tailed $t$ test; $t=1.4 ; p>0.05$ ) was not influenced by $\mathrm{B} 51$ depolarization. Resting membrane potentials (in $\mathrm{mV}$ ): $A$ : $(\mathrm{CBI}-4,-63 ; \mathrm{B} 51,-57$; $\mathrm{C}-\mathrm{B} 51,-63$; $B 8,-59 ; B: C B I-4,-60 ; B 64,-74 ; B 51,-66 ; C-B 51,-61 ; C: C B I-4,-60 ; B 51,-64 ; C-B 51$, $-65 ; B 8,-62$.

and may induce plateau potentials in B64, thereby initiating a switch from protraction to retraction.

We also examined other connections of CBI- 4 and B30. CBI-4 elicited fast monosynaptic EPSPs in three protraction interneu- rons: $\mathrm{B} 30(n=15), \mathrm{B} 63(n=4)$, and $\mathrm{B} 20(n=6)$ (Fig. $7 A)$. The amplitude of the fast EPSP was typically largest in B63 and smallest in B20. In addition, B30 was electrically coupled to B63 (coupling ratio from $\mathrm{B} 63$ to $\mathrm{B} 30,0.14$; from $\mathrm{B} 30$ to $\mathrm{B} 63,0.08$ ) because negative current injection in $\mathrm{B} 63$ induced a hyperpolarizing response in B30 both in normal saline (Fig. $7 B)(n=4)$ and in high divalent saline (data not shown). Finally, B30 received fast IPSPs from the retraction-interneuron B64 (Fig. $7 B)(n=7)$.

Taken together, synaptic inputs and outputs of B30 explain how $\mathrm{B} 30$ promotes $\mathrm{B} 8$ firing during retraction, inhibits $\mathrm{B} 8$ firing during protraction, and promotes a short duration of protraction, and finally how B30 itself is activated during protraction.

Role of B51 in the second B8 burst during hyper-retraction To determine the modular basis of the generation of the second B8 burst, we studied B64 and B51, buccal neurons that are normally active during retraction and hyper-retraction. B64 is a retraction-phase interneuron that switches motor programs from protraction to retraction (Hurwitz and Susswein, 1996). B51 is a proprioceptor that also functions as an interneuron (Plummer and Kirk, 1990; Evans and Cropper, 1998; Nargeot et al., 1999). Both B64 and B51 are neurons that generate plateaulike potentials, but they show different activity patterns in CBI4-elicited motor programs that are accompanied by a second B8 burst-hyper-retraction. Although B64 was active throughout the retraction and hyper-retraction phase, B51 was normally active only during hyper-retraction (Fig. 8). Specifically, although B64 was immediately activated after initiation of the retraction phase, B51 did not spike in the early part of retraction. Instead, B51 slowly depolarized as retraction progressed. Thus, when the buildup of depolarization in B51 led to spiking, the second B8 burst was invariably observed. In contrast, when a second B8 burst was not observed, B51 would repolarize, and the buildup of depolarization would promptly terminate with little or no spiking in B51. Thus, B51 activity is correlated with the second $\mathrm{B} 8$ burst.

To determine whether B51 activity is necessary and sufficient for the second B8 burst, we performed hyperpolarization-depolarization experiments. In preparations in which CBI-4 evoked programs with a second B8 burst, hyperpolarization of B51 prevented the second B8 burst from occurring when the original programs were either ingestive (Fig. $8 A)(n=3)$ or egestive (Fig. $8 B)(n=4)$. Conversely, in preparations in which CBI-4 evoked programs without a second B8 burst, depolarization of B51 activated the second B8 burst (Fig. $8 C)(n=3)$. Notice that despite the fact that current was injected earlier during retraction (Fig. $8 C)$, B51 was not activated until later during retraction, at about the time when the retraction would have ended without a second B8 burst. The mechanisms that underlie the delayed onset of B51 burst during retraction have not been identified. One possibility is that B51 receives inhibition from neurons that are active in the early part of retraction but are not active during hyper-retraction. Independent of the mechanisms that may underlie the delayed onset of B51 firing, these experiments indicate that B51 is indeed important for the second B8 burst.

Furthermore, when CBI-2 elicited motor programs without a second $\mathrm{B} 8$ burst, activation of $\mathrm{B} 51$ also induced $\mathrm{B} 8$ activity resembling a second B8 burst (Fig. 9A). To provide quantitative evidence supporting the addition of the second B8 burst during B51 activation, we analyzed the duration of the B8 burst during retraction in episodes before, during, and after B51 stimulation in these experiments (Fig. 9B) $(n=5)$. When B51 was stimulated, the retraction phase was divided into two parts, the initial retraction without B51 activity and the remaining retraction with B51 


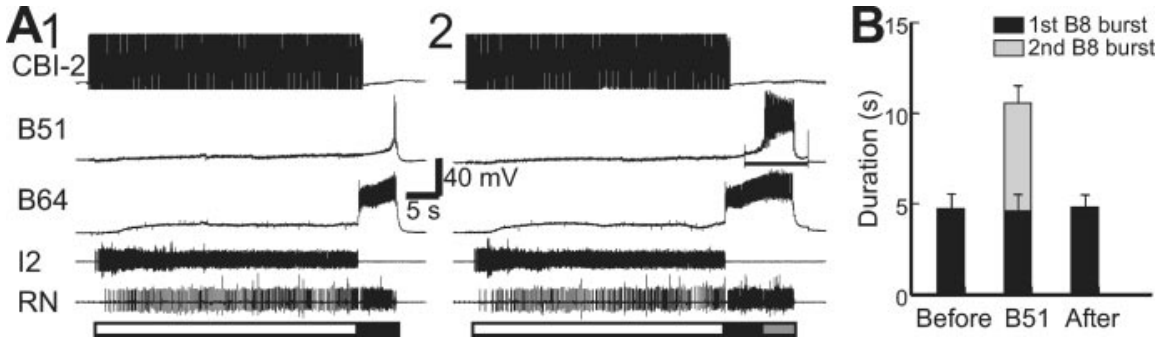

Figure 9. B51 induces activity resembling the second $\mathrm{B} 8$ burst in $\mathrm{CBI}-2$-elicited motor programs. $A, \mathrm{CBI}-2$ stimulation elicited an ingestive program without a second B8 burst (as monitored by activity in RN) (A1), as is normally the case for (BI-2-elicited programs. Current injection into B51 (bar) induced B51 firing and a second B8 burst (gray bar) at the end of retraction (A2). Notice that $B 64$ was active throughout retraction and hyper-retraction (the second $B 8$ burst). $B$, Group data $(n=5)$ of retraction duration during (BI-2-elicited ingestive programs in episodes before, during, and after B51 stimulation. In cycles during which B51 was stimulated, we measured the duration of B8 activity in the absence of B51 activity (black bar; presumably the first B8 burst, $4.6 \pm$ $0.9 \mathrm{sec}$ ) and B8 activity in the presence of B51 activity (gray bar; presumably the second B8 burst, $5.9 \pm 0.9 \mathrm{sec}$ ). We performed statistical analyses on the data and found that when the durations of B8 activity in before $(4.8 \pm 0.8 \mathrm{sec})$ and after $(4.9 \pm 0.7 \mathrm{sec})$ groups was compared with the duration of the first B8 burst in episodes with B51 stimulation, there was no statistically significant difference among these group data (repeated-measures ANOVA; $F_{(2,8)}=0.4 ; p>0.05$ ). In contrast, when durations of the first and second B8 burst in cycles with B51 stimulation were added together (10.6 $\pm 1.1 \mathrm{sec}$ ) and then compared with those in the before and after groups, there was a statistically significant difference among these group data [repeated-measures ANOVA; $F_{(2,8)}=31.3 ; p<0.001 ;$ a Bonferroni multiple comparisons test of different group pairs showed that the total retraction duration was significantly different from that in the before $(t=6.9 ; p<0.001)$ and after $(t=6.8 ; p<0.001)$ groups, whereas the retraction duration in the before group was not significantly different from that in the after group $(t=0.1 ; p>0.05)]$, suggesting that B51 indeed caused activation of the additional B8 activity that can be considered as the second B8 burst. Resting membrane potentials (in $\mathrm{mV}$ ): (BI-2, -62; B51, -68; B64, -73 .

activity. These data are stacked together with the initial retraction at the bottom (Fig. 9B, black bar) and the remaining retraction at the top (gray bar). Statistical analyses (Fig. 9, legend) indicate that the second part of retraction during B51 activation is distinct from the initial retraction and resembles the second B8 bursthyper-retraction. Thus, these findings further support the idea that the second B8 burst is likely to be a phase that is distinct from retraction, because B51 is only active during this phase and is both necessary and sufficient for its occurrence.

The role of B51 in mediating the second B8 burst is consistent with the pattern of synaptic connections that B51 makes in feeding circuits. B51 connections have been widely described in previous reports (Plummer and Kirk, 1990; Evans and Cropper, 1998; Nargeot et al., 1999). B51 has been shown to provide fast EPSPs to B8, thus explaining its ability to activate the second B8 burst. B51 is also electrically coupled to neurons that constitute the retraction circuitry, such as B64.

\section{Discussion}

We use Aplysia feeding as a model system for studying how complex behaviors are generated by interneuronal networks. Specifically, we build on previous suggestions that complex behaviors are constructed through flexible combinations of modules and address two major questions. What may constitute a module? Are there different types of modules? The Aplysia feeding system is advantageous for such an endeavor because the underlying motor network is relatively simple. Yet the three studied forms of behavior, biting, swallowing, and rejection, are complex behaviors in that the motor apparatus, known as the radula, generates more than one type of movement: protraction-retraction versus opening-closing. Distinct behaviors are produced by changing coordination patterns of sets of movements and by changing the duration of specific phases of activity.

Previously (Jing and Weiss, 2001, 2002), we studied biting and rejection-like motor programs elicited by the higher-order interneuron CBI-2. We examined how the different coordination patterns of radula closure relative to protraction-retraction are generated. These data were consistent with a modular organization in the feeding interneuronal network. Because in our previous paper (Jing and Weiss, 2002) we focused on examining how motoneuronal activity is controlled by interneurons, we referred to interneurons as movement controllers. In the present work, we show that individual interneurons are multifunctional, and we now opt for a more general term, modules, and thus follow the convention in the vertebrate literature (Bizzi et al., 1991; Stein et al., 1995). Furthermore, by examining the motor programs elicited by an additional CBI, we identified additional types of motor programs that resemble additional forms of feeding in Aplysia (Morton and Chiel, 1993a). This enabled us to examine several issues relevant to modular organization that were not addressed in the previous work.

In general, a module has been defined as a discrete entity with a function that can be separated from the function of other entities (Hartwell et al., 1999). In Aplysia, interneurons that are critical for protraction-retraction (B63 and B64) (Hurwitz and Susswein, 1996; Hurwitz et al., 1997), appear to be active in both biting and rejection-like programs. In contrast, the interneurons that determine the phasing of radula closure activity differ for the two programs (B40 used during biting and B20 during egestion). Thus B63 and B64 may be basic and obligatory building blocks (modules) for the generation of behaviors (Fig. $10 \mathrm{~A}$ ), whereas $\mathrm{B} 40$ and $\mathrm{B} 20$ are modules that are responsible for a specific feature of a behavior.

Although the above findings support the notion that the feeding network has a modular organization, other issues regarding the composition of a module remained unresolved. Specifically, it was proposed (Bernstein, 1967; Tresch et al., 2002) that a module may function to reduce the number of degrees of freedom by controlling groups of muscles that are used to mediate features common to different behaviors and thus may be behavior independent. In contrast, a module may control multiple salient features of a behavior and thus may act to specify a behavior. From this perspective, B63 and B64 are examples of behaviorindependent modules, because they are active in all motor programs, including biting, swallowing, and rejection. Before this study, however, it was not clear whether B40 and B20 are examples of behavior-specific modules. For example, biting and swallowing differ in the duration of protraction but are similar in that radula closure occurs primarily during retraction. The latter feature is determined by B40 during biting. The question then was whether B40 also mediates closure phasing for swallowing. If it did, B40 should be considered a behavior-independent module; otherwise, B40 would be a behavior-specific module.

Here, we identified swallowing-like motor programs that are elicited by another higher-order interneuron, CBI-4, and we found the latter scenario to be true. Closure phasing is not determined by B40; instead it is determined by a novel interneuron, B30. Thus, these data support the idea that behavior-specific modules generate distinct but related motor programs (Fig. $10 \mathrm{~B})$. Consistent with this notion, in swallowing, B30 controls 


\section{Modular organization of Aplysia feeding motor network A Basic setup (kernel) of $\begin{gathered}\text { B63 network } \\ \text { the net }\end{gathered}$}

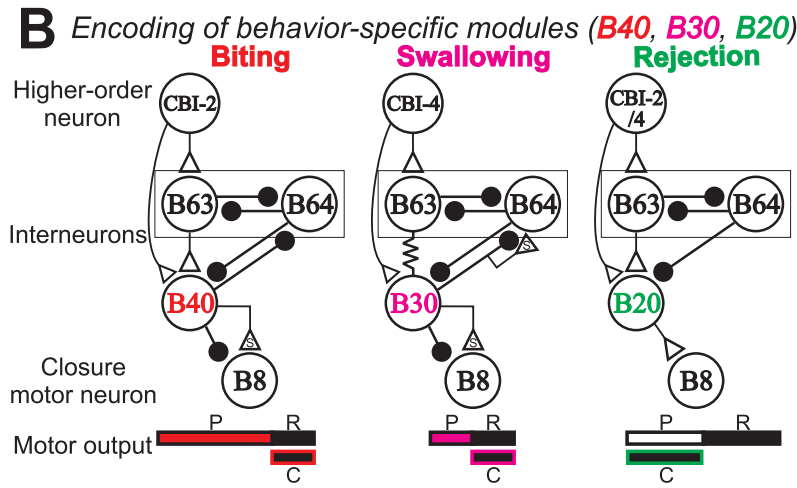

C Encoding of a behavior-independent module (B51)

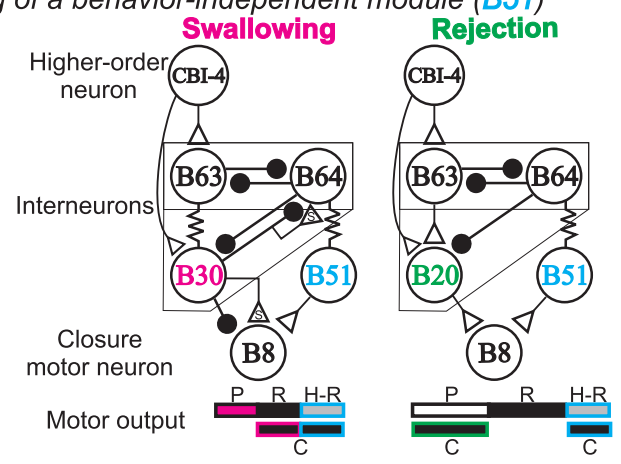

Figure 10. Circuit diagrams illustrating the construction of distinct motor programs through combinations of behavior-specific and behavior-independent modules. For clarity, we show different functional configurations that include only neurons with activity that predominates (for details, see Fig. 1). A, The initiation of motor programs occurs when the protraction interneuron B63 is activated by the higher order interneurons, (BI-2 (Hurwitz et al., 2003) or CBI-4. B63 and the retraction-interneuron B64 reciprocally inhibit each other and mediate the alternating protraction $(P)$-retraction $(R)$ cycles. Because they are active in all motor programs, both B63 and B64 are behavior-independent modules. B, The critical parameters for bitingswallowing-, and rejection-like programs are encoded by three separate behavior-specific modules: $B 40, B 30$, and $B 20$. B40 appears to be responsible for $B 8(C)$ activity phasing during retraction and long protraction duration for biting. $B 30$ appears to be responsible for $B 8(C)$ activity phasing during retraction and short protraction duration for swallowing. B20 appears to be responsible for $B 8(C)$ activity phasing during protraction for rejection. $C, A$ behaviorindependent module, B51, encodes the second B8 burst (hyper-retraction) (H-R) that can be flexibly added to a subset of swallowing- and rejection-like programs elicited by CBI-4. The interneurons and the parameters that they encode are color-coded. Open triangle, Excitation; closed circle, inhibition; s, slow synaptic actions; zig-zagged line, electrical coupling.

protraction duration, in addition to closure phasing, as B40 does in biting (Jing and Weiss, 2002; Jing et al., 2003). Furthermore, for the shared parameter, B30 uses a similar synaptic mechanism (fast inhibition and slow excitation) to mediate closure phasing as B40 does. For the parameter that differs between biting and swallowing (protraction duration), B30 uses weak fast inhibition and a prominent slow excitation of B64 to activate it with a short delay and shorten protraction, whereas B40 uses strong fast inhibition in $\mathrm{B} 64$ to extend protraction. Thus, it may be common that behavior-specific modules encode more than one parameter that are critical for a behavior.

Interestingly, we also found evidence for a type of a behaviorindependent module (B51) that mediates one distinct feature of CBI-4-elicited programs but differs from other behavior- independent modules shown previously (B63, B64). Specifically, we observed a second closure burst (hyper-retraction) that can be flexibly added to the end of CBI-4-elicited programs, regardless of the type of program that it is. We showed that the interneuron B51 contributes significantly to this phase (Fig. 10C). If we consider B63 and B64 as a type of behavior-independent module that mediates protraction-retraction in all programs, B51 clearly represents a different type of module that may be used in some programs but not others. The differential function of these two types of behavior-independent modules is evident, because protraction and retraction are critical for the expression of a basic behavior regardless of its type, whereas hyper-retraction is optional and may be related to the presence of external factors. Indeed, B51 has been found to be a proprioceptor that is sensitive to the amount of resistance that is encountered as the radula retracts (Evans and Cropper, 1998). Such sensory inputs in swallowing may signal the successful grasp of food, and hyperretraction may be used to overcome resistance and draw food deeper into the esophagus. Similarly, the flexible add-on of muscle activity has been described for the frog wipe reflex during circumvention of path obstacles (Kargo and Giszter, 2000). Notably, in this paper, we often identified single neurons as one module, with the exception of B30, which has two members; however, we cannot exclude the possibility that some of the modules may contain additional members. One way in which additional neurons could be involved is through electrical coupling that is present in the network.

The present work indicates that the concept of modularity is more complex than was originally conceived (Grillner, 1981; Bizzi et al., 1991; Stein and Smith, 1997; Jing and Weiss, 2002; Tresch et al., 2002). Here, by directly examining the functions of interneurons, we have delineated diverse types of modules that are used to construct behaviors (Fig. 10). In particular, our study establishes the importance of behavior-specific modules in encoding multiple salient features of a behavior. The use of behavior-specific modules has three implications. First, this encoding scheme may simplify the selection of behavior by higherorder interneurons-sensory inputs, which may recruit a smaller number of neurons when switching from one form of behavior to another. In human motor learning, because of the abundance of contexts within which we must act, multiple controllers (modules) rather than single ones (Wolpert and Kawato, 1998; MussaIvaldi, 1999; Wolpert and Ghahramani, 2000) have been proposed to coexist, with each module suitable for one or a small set of contexts. This organization is also advantageous in that it allows individual modules to participate in motor learning without affecting motor behaviors that are mediated by other modules (Wolpert and Kawato, 1998). We propose that these modules in human motor control are developed from the behavior-specific modules similar to the ones described here, with higher-order interneurons and other sensory interneurons channeling appropriate contexts to these modules. Second, joint control of multiple parameters by a module may be advantageous when these parameters of a behavior covary. For example, a long protraction in biting likely requires strong inhibition from B40 to the closure motoneuron B8 to counter slow excitation from B40 that is building up during protraction. In swallowing, protraction is short, and therefore this is unnecessary. Indeed, we found that fast inhibition from B40 to B8 appears to be stronger than that from $\mathrm{B} 30$ to $\mathrm{B} 8$. Third, for the sake of discussion, we have emphasized the behavioral specificity of modules. We did not mean to imply, however, that this specificity is all or none. In fact, our data suggest that behavior-specific modules often do not act in an 
all-or-none manner, but instead tend to promote the generation of behaviors that have certain features. Thus, these behaviorspecific modules can actually be combined to generate additional forms of motor patterns, e.g., intermediate programs. Importantly, the ability to combine the actions of behavior-specific modules that promote different types of behaviors may provide a basis for additional behavioral flexibility. This view of the modular control of behavior bears a certain formal similarity to the notion of population coding (Berkowitz and Stein, 1994; Sparks et al., 1997; Nicolelis, 2001) and may thus integrate the concepts of modular organization and population coding in motor control.

In conclusion, our data suggest that complex motor acts are constructed using two broad classes of module that may fulfill complementary functions. Whereas behavior-specific modules serve to define the nature of behavior for a specific goal, the use of behavior-independent modules represents an efficient and economic strategy for the execution of complex behaviors.

\section{References}

Berkowitz A, Stein PS (1994) Activity of descending propriospinal axons in the turtle hindlimb enlargement during two forms of fictive scratching: broad tuning to regions of the body surface. J Neurosci 14:5089-5104.

Bernstein N (1967) The coordination and regulation of movements. Oxford: Pergamon.

Bizzi E, Mussa-Ivaldi FA, Giszter S (1991) Computations underlying the execution of movement: a biological perspective. Science 253:287-291.

Church PJ, Lloyd PE (1994) Activity of multiple identified motor neurons recorded intracellularly during evoked feeding-like motor programs in Aplysia. J Neurophysiol 72:1794-1809.

Cohen JL, Weiss KR, Kupfermann I (1978) Motor control of buccal muscles in Aplysia. J Neurophysiol 41:157-180.

Cropper EC, Kupfermann I, Weiss KR (1990) Differential firing patterns of the peptide-containing cholinergic motor neurons B15 and B16 during feeding behavior in Aplysia. Brain Res 522:176-179.

d'Avella A, Saltiel P, Bizzi E (2003) Combinations of muscle synergies in the construction of a natural motor behavior. Nat Neurosci 6:300-308.

Dembrow NC, Jing J, Proekt A, Romero A, Vilim FS, Cropper EC, Weiss KR (2003) A newly identified buccal interneuron initiates and modulates feeding motor programs in Aplysia. J Neurophysiol 90:2190-2204.

Evans CG, Cropper EC (1998) Proprioceptive input to feeding motor programs in Aplysia. J Neurosci 18:8016-8031.

Grasso R, Bianchi L, Lacquaniti F (1998) Motor patterns for human gait: backward versus forward locomotion. J Neurophysiol 80:1868-1885.

Grillner S (1981) Control of locomotion in bipeds, tetrapods, and fish. In: Handbook of physiology, Sect 1, Vol 2 (Brooks V, ed), pp 1179-1236. Bethesda, MD: American Physiological Society.

Grillner S (1985) Neurobiological bases of rhythmic motor acts in vertebrates. Science 228:143-149.

Hartwell LH, Hopfield JJ, Leibler S, Murray AW (1999) From molecular to modular cell biology. Nature 402:C47-52.

Hurwitz I, Susswein AJ (1996) B64, a newly identified central pattern generator element producing a phase switch from protraction to retraction in buccal motor programs of Aplysia californica. J Neurophysiol 75:1327-1344.

Hurwitz I, Neustadter D, Morton DW, Chiel HJ, Susswein AJ (1996) Activity patterns of the B31/B32 pattern initiators innervating the I2 muscle of the buccal mass during normal feeding movements in Aplysia californica. J Neurophysiol 75:1309-1326.

Hurwitz I, Kupfermann I, Susswein AJ (1997) Different roles of neurons B63 and B34 that are active during the protraction phase of buccal motor programs in Aplysia californica. J Neurophysiol 78:1305-1319.

Hurwitz I, Kupfermann I, Weiss KR (2003) Fast synaptic connections from CBIs to pattern-generating interneurons in Aplysia: initiation and modification of motor programs. J Neurophysiol 89:2120-2136.

Jing J, Weiss KR (2001) Neural mechanisms of motor program switching in Aplysia. J Neurosci 21:7349-7362.

Jing J, Weiss KR (2002) Interneuronal basis of the generation of related but distinct motor programs in Aplysia: implications for current neuronal models of vertebrate intralimb coordination. J Neurosci 22:6228-6238.
Jing J, Vilim FS, Wu JS, Park JH, Weiss KR (2003) Concerted GABAergic actions of Aplysia feeding interneurons in motor program specification. J Neurosci 23:5283-5294.

Jordan LM (1991) Brainstem and spinal cord mechanisms for the initiation of locomotion: single limb analysis. In: Neurobiological basis of human locomotion (Shimamura M, Grillner S, Edgerton VR, eds), pp 3-20. Tokyo: Japan Scientific Societies.

Kargo WJ, Giszter SF (2000) Rapid correction of aimed movements by summation of force-field primitives. J Neurosci 20:409-426.

Krzanowski WJ, Lai YT (1988) A criterion for determining the number of groups in a data set using sum-of-squares clustering. Biometrics 44:23-34.

Kupfermann I (1974) Feeding behavior in Aplysia: a simple system for the study of motivation. Behav Biol 10:1-26.

Marriott FH (1971) Practical problems in a method of cluster analysis. Biometrics 27:501-514.

Morgan PT, Jing J, Vilim FS, Weiss KR (2002) Interneuronal and peptidergic control of motor pattern switching in Aplysia. J Neurophysiol $87: 49-61$.

Mortin LI, Keifer J, Stein PS (1985) Three forms of the scratch reflex in the spinal turtle: movement analyses. J Neurophysiol 53:1501-1516.

Morton DW, Chiel HJ (1993a) In vivo buccal nerve activity that distinguishes ingestion from rejection can be used to predict behavioral transitions in Aplysia. J Comp Physiol 172:17-32.

Morton DW, Chiel HJ (1993b) The timing of activity in motor neurons that produce radula movements distinguishes ingestion from rejection in Aplysia. J Comp Physiol 173:519-536.

Mussa-Ivaldi FA (1999) Modular features of motor control and learning. Curr Opin Neurobiol 9:713-717.

Nargeot R, Baxter DA, Byrne JH (1999) In vitro analog of operant conditioning in Aplysia. II. Modifications of the functional dynamics of an identified neuron contribute to motor pattern selection. J Neurosci 19:2261-2272.

Nicolelis MAL, ed (2001) Advances in neural population coding. Amsterdam: Elsevier.

Plummer MR, Kirk MD (1990) Premotor neurons B51 and B52 in the buccal ganglia of Aplysia californica: synaptic connections, effects on ongoing motor rhythms, and peptide modulation. J Neurophysiol 63:539-558.

Rosen SC, Teyke T, Miller MW, Weiss KR, Kupfermann I (1991) Identification and characterization of cerebral-to-buccal interneurons implicated in the control of motor programs associated with feeding in Aplysia. J Neurosci 11:3630-3655.

Sparks DL, Kristan WB, Shaw BK (1997) The role of population coding in the control of movement. In: Neurons, networks, and motor behavior (Stein PS, Grillner S, Selverston AI, Stuart DG, eds), pp 21-32. Cambridge, MA: MIT.

Stein PS, Smith JL (1997) Neural and biomechanical control strategies for different forms of vertebrate hindlimb motor tasks. In: Neurons, networks, and motor behavior (Stein PSG, Grillner S, Selverston AI, Stuart DG, eds), pp 61-73. Cambridge, MA: MIT.

Stein PS, Daniels-McQueen S (2002) Modular organization of turtle spinal interneurons during normal and deletion fictive rostral scratching. J Neurosci 22:6800-6809.

Stein PS, Victor JC, Field EC, Currie SN (1995) Bilateral control of hindlimb scratching in the spinal turtle: contralateral spinal circuitry contributes to the normal ipsilateral motor pattern of fictive rostral scratching. J Neurosci 15:4343-4355.

Tresch MC, Saltiel P, Bizzi E (1999) The construction of movement by the spinal cord. Nat Neurosci 2:162-167.

Tresch MC, Saltiel P, d'Avella A, Bizzi E (2002) Coordination and localization in spinal motor systems. Brain Res Brain Res Rev 40:66-79.

Trudeau LE, Castellucci VF (1992) Contribution of polysynaptic pathways in the mediation and plasticity of Aplysia gill and siphon withdrawal reflex-evidence for differential modulation. J Neurosci 12:3838-3848.

Weiss KR, Chiel HJ, Koch U, Kupfermann I (1986) Activity of an identified histaminergic neuron, and its possible role in arousal of feeding behavior in semi-intact Aplysia. J Neurosci 6:2403-2415.

Wolpert DM, Ghahramani Z (2000) Computational principles of movement neuroscience. Nat Neurosci [Suppl] 3:1212-1217.

Wolpert DM, Kawato M (1998) Multiple paired forward and inverse models for motor control. Neural Net 11:1317-1329. 